\title{
Discrimination and Content Analysis of Fritillaria Using Near Infrared Spectroscopy
}

\author{
Yu Meng, ${ }^{1,2}$ Shisheng Wang, ${ }^{1,2}$ Rui Cai,, ${ }^{1,2}$ Bohai Jiang, ${ }^{1,2}$ and Weijie Zhao ${ }^{1,2}$ \\ ${ }^{1}$ State Key Laboratory of Fine Chemicals, Dalian University of Technology, Dalian 116024, China \\ ${ }^{2}$ School of Pharmaceutical Science and Technology, Dalian University of Technology, Dalian 116024, China \\ Correspondence should be addressed to Weijie Zhao; zyzhao@dlut.edu.cn
}

Received 3 January 2015; Revised 2 February 2015; Accepted 2 February 2015

Academic Editor: Miguel de la Guardia

Copyright (c) $2015 \mathrm{Yu}$ Meng et al. This is an open access article distributed under the Creative Commons Attribution License, which permits unrestricted use, distribution, and reproduction in any medium, provided the original work is properly cited.

\begin{abstract}
Fritillaria is a traditional Chinese herbal medicine which can be used to moisten the lungs. The objective of this study is to develop simple, accurate, and solvent-free methods to discriminate and quantify Fritillaria herbs from seven different origins. Near infrared spectroscopy (NIRS) methods are established for the rapid discrimination of seven different Fritillaria samples and quantitative analysis of their total alkaloids. The scaling to first range method and the partial least square (PLS) method are used for the establishment of qualitative and quantitative analysis models. As a result of evaluation for the qualitative NIR model, the selectivity values between groups are always above 2 , and the mistaken judgment rate of fifteen samples in prediction sets was zero. This means that the NIR model can be used to distinguish different species of Fritillaria herbs. The established quantitative NIR model can accurately predict the content of total alkaloids from Fritillaria samples.
\end{abstract}

\section{Introduction}

The plant Fritillaria belongs to the genus Fritillaria of the Liliaceae family (known in English as fritillary). This family is native to the temperate regions of the Northern Hemisphere [1]. The Fritillaria bulb has many medical uses, especially in traditional Chinese medicine, such as clearing heat, moistening lung, eliminating phlegm, and relieving cough, and for the treatment of mammary abscess, pulmonary dryness, and chronic cough [2]. The genus Fritillaria includes about 130 species worldwide. The different species each have unique effects. Bulbus Fritillariae Cirrhosae and F. thunbergii Miq. are widely used in China for different purposes. F. thunbergii Miq. is good for detoxification, eliminating phlegm, releasing stagnated lung-qi, and treating "wind-fire syndrome" in traditional Chinese medicine. Bulbus Fritillariae Cirrhosae has a sweet flavor and is used for lung tonic and treatment of cough. In northeast and northwest of China, Bulbus Fritillariae Cirrhosae is always replaced by F. pallidiflora and F. ussuriensis as a medication. Because of its various subspecies and diverse effects, Fritillaria is easily confused and misused (shown in Figure 1). Most of the species are similar in morphology, making it hard to distinguish one from another. Since the existing identification methods are inconvenient and troublesome, developing a fast, simple, accurate, and solvent-free method to discriminate the varieties of Fritillaria is very useful.

Steroidal alkaloids are the main bioactive constituents in genus Fritillaria; the content of total alkaloids is the major index of quality evaluation of Fritillaria materials. The type and content of alkaloids differ among species. BulbusFritillariae Thunbergii mainly contains peimine, peiminine, sipeimine, peimisine, and peimiphine [3]; Bulbus Fritillariae Cirrhosae contains fritimine, minpeimine, peiminine, sonpeimine, chinpeimine, and sipeimine [4]; Bulbus Fritillariae Pallidiflorae contains fritimine, sipeimine, peimisine, and oxynitrides of sipeimine [5]. Among the steroidal alkaloids in fritillaries, peiminine and sipeimine exist in every species of Fritillaria and can thus be selected as a standard to determine the total alkaloid content (Figure 2). The main measurement methods include the ultraviolet spectrum $[4,5]$, ELSD [6], and colorimetry [7].

Near infrared (NIR) light refers to the electromagnetic waves located between visible light and intermediate infrared 


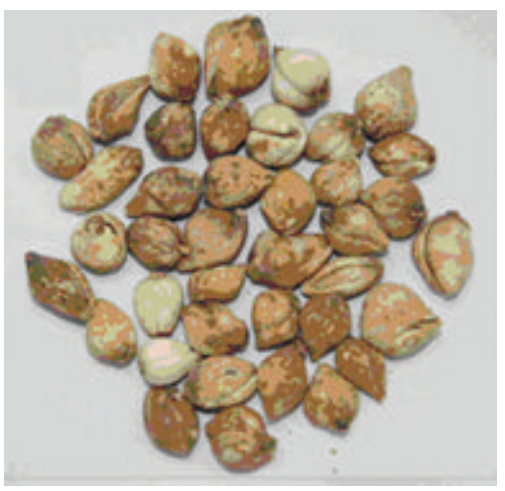

(a)

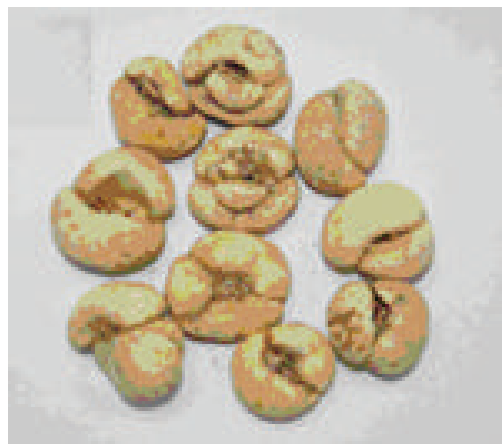

(d)

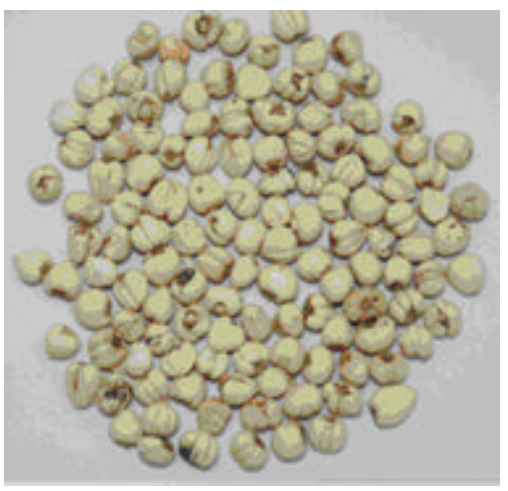

(b)

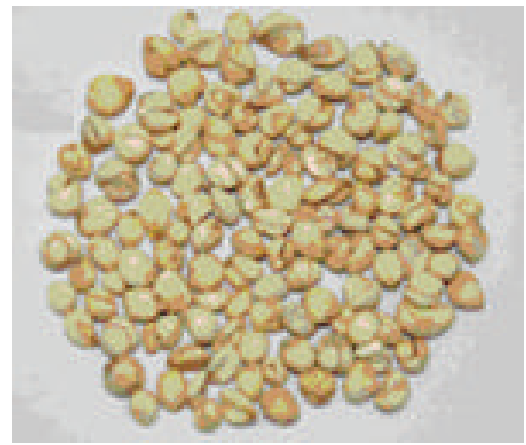

(e)

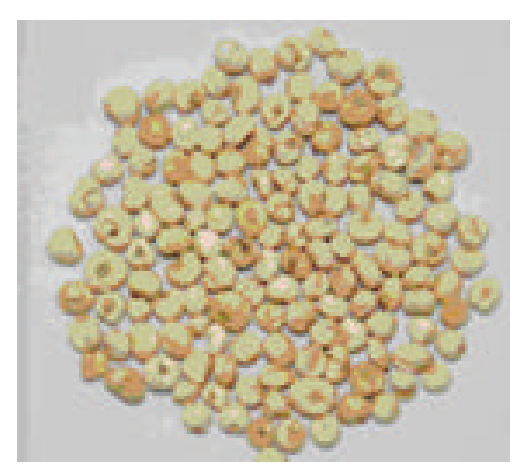

(g)

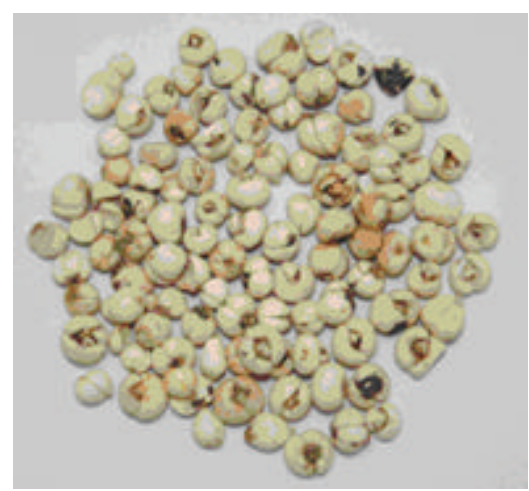

(c)

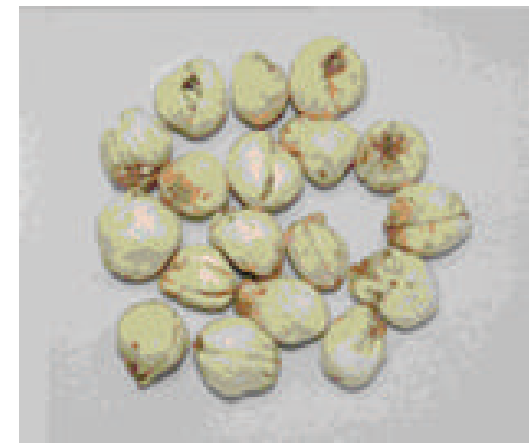

(f) 

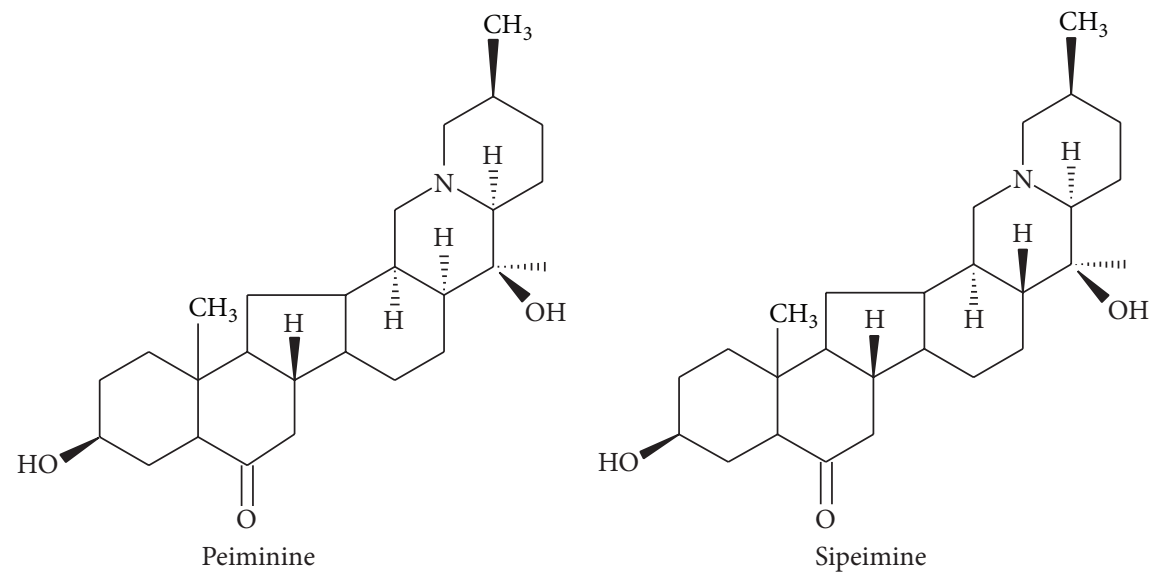

FIGURE 2: Structures of peiminine and sipeimine.

Province), F. cirrhosa D. Don (Sichuan Province), F. thunbergii Miq. (big) (Zhejiang Province), F. thunbergii Miq. (small) (Zhejiang Province), F. pallidiflora Schrenk (Xinjiang Province), and F. ussuriensis Maxim (Heilongjiang Province) were collected from the place of origin in 2007 and authenticated by Daixian Chen who is a chief pharmacist of traditional Chinese medicine from Dalian Institute for Food and Drug Control.

2.1.2. Instruments. The Bruker NIR Spectrometer (MPA; Bruker Optics, Germany), equipped with an indium gallium arsenide (InGaAs) and a lead sulfide ( $\mathrm{PbS}$ ) detector, was used in the experiments; Unicam Helios Alpha 9423 UV Vis Double Beam Spectrophotometer (UNICAM) and Mettler Toledo AG245 Dual Range Analytical Scale Balance (Mettler) were also used.

2.1.3. Reagent. Methanol (HPLC grade, Merck), ultrapure water (Millipore), chloroform (Merck) and potassium biphthalate (Merck) were used. The standard substances, peiminine (20 mg, batch number 110751-200607) and sipeimine (20 mg, batch number 110767-200504), were both purchased from National Institutes for Food and Drug Control.

2.2. Sample Pretreatment. The seven Fritillaria samples were crushed separately and then ground into fine powder. Each powder was sieved on a 100 mesh and dried under $60^{\circ} \mathrm{C}$ for $12 \mathrm{~h}$ to yield a $6 \mathrm{~g}$ sample. The seven samples were reserved in a dry atmosphere.

\subsection{Content Determination of the Total Alkaloids by UV Spectroscopy}

2.3.1. Determination of Standard Curve. About $2.0 \mathrm{mg}$ of standard peiminine was dissolved in $100 \mathrm{~mL}$ chloroform, and the standard peiminine solutions of 1.0, 2.0, 3.0, 4.0, and $5.0 \mathrm{~mL}$ were set in $60 \mathrm{~mL}$ separating funnels, respectively. Chloroform was then added to $8.0 \mathrm{~mL}$, before $2.0 \mathrm{~mL}$ of $\mathrm{pH}$
5 buffer solution and $2.0 \mathrm{~mL}$ of $0.001 \mathrm{~mol} / \mathrm{L}$ bromothymol blue were added. After shaking and standing, the chloroform layer was filtered, and the aqueous part was washed with chloroform. The combined chloroform solution was diluted to $10 \mathrm{~mL}$, and the absorbance was measured at $410 \mathrm{~nm}$ with blank control as a reference.

The linear regression equation was obtained as follows:

$$
A=0.000541+0.040201 C \quad(r=0.9998) .
$$

2.3.2. Preparation of Total Alkaloids from Fritillaries. Seven samples of different fritillary powders, each weighing about $2 \mathrm{~g}$, were wetted with $18 \%$ ammonia water $2 \mathrm{~mL}$ for $1 \mathrm{~h}$ in the conical flask with a plug and then extracted twice with a mixed solvent (diethyl ether/chloroform/95\% ethanol $25: 8: 2.5)$ in ultrasonic cleaner $(30$ and $15 \mathrm{~mL}$, resp., for $30 \mathrm{~min}$ each time). After washing residues with $15 \mathrm{~mL}$ of mixed solvent, the combined solution was evaporated to dryness at $60^{\circ} \mathrm{C}$ and then dissolved with chloroform to $10 \mathrm{~mL}$ for analysis.

2.3.3. Content Determination of Total Alkaloids from Fritillaries. Each sample of total alkaloid solution $0.8 \mathrm{~mL}$ was evaporated to dryness, and chloroform $8.0 \mathrm{~mL}$, a pH 5 buffer solution of $2.0 \mathrm{~mL}, 0.001 \mathrm{~mol} / \mathrm{L}$ of bromothymol blue $2.0 \mathrm{~mL}$ were added. After a severe shake and stationary placement, the chloroform layer was filtered, and the aqueous layer was washed by chloroform. The combined chloroform solution was diluted to $10 \mathrm{~mL}$. The absorbance of the sample at $410 \mathrm{~nm}$ was measured immediately.

UV absorption regression equation of peiminine was obtained as follows:

$$
A=0.000541+0.040201 C \quad(r=0.9998) .
$$

The total alkaloid content in Fritillaria can be calculated by formula (3), where $A$ is the UV absorbance at $410 \mathrm{~nm}, N$ is the dilution times 125 , and $W$ is the sample weight $(\mathrm{g})$ :

$$
\text { Total alkaloids }(\%)=\frac{(A-0.000541)}{W \times 106 \times 0.040201} \times N \text {. }
$$




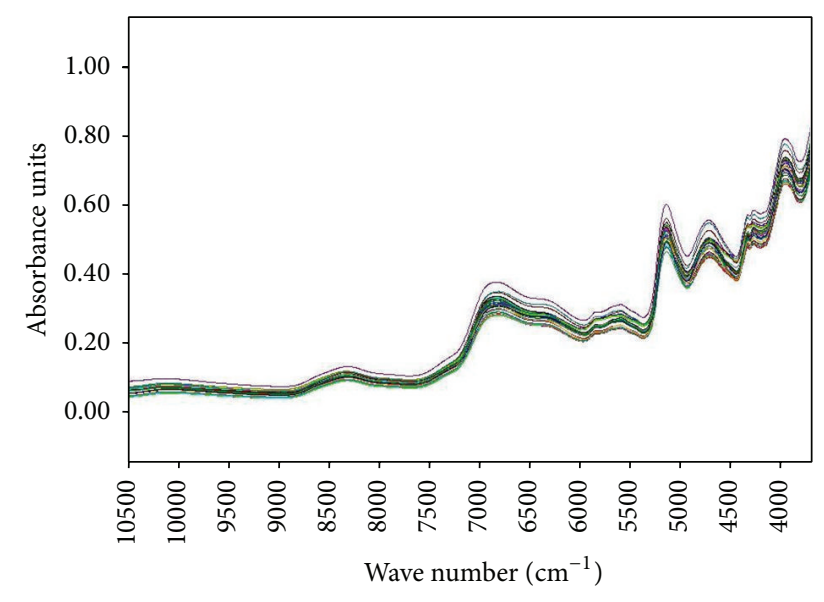

FIGURE 3: The original NIR spectrum of seven Fritillaria samples.

\subsection{NIR Spectrum Collection. Consider the following.}

Instrument condition: spectral resolution, $4 \mathrm{~cm}^{-1}$. Sample scanning times: 32. Background scanning times: 32 . Scanning range: $12000 \sim 3700 \mathrm{~cm}^{-1}$.

Procedure: put some powder in the measurement glass. The original spectrum of each sample is collected by using diffuse reflection method for 3 times and the average spectrum is obtained. The NIR spectra of seven Fritillaria samples are as follows (Figure 3).

2.5. Data Analysis. The calibration models of quantitative and qualitative analysis were developed by OPUS 6.5 software from Bruker Optics and MATLAB 7.1 software. For quantitative analysis, a PLS regression and a leave-one-out (LOO) cross validation were used to construct calibration models. The performance of the calibration models was evaluated by comparing the NIR predicted values with the UV measured values and evaluating parameters including the root means square error of cross validation (RMSECV), root mean square error of prediction (RMSEP), and correlation coefficient $\left(R^{2}\right)$. For the validation set, square error of prediction (SEP) was used to verify the precision of the developed NIRS model. Detailed computing formula of above mentioned parameters can be found in [16].

The identification model was established by utilizing algorithms such as factorization and scaling to first range, which were provided by OPUS 6.5. Factor analysis was carried out on the spectra, and the spectrum $(a)$ can be expressed as a linear combination of orthogonal factor spectra $\left(f_{i}\right)$ and $T_{i}$ : $a=T_{1 a} f_{1}+T_{2 a} f_{2}+T_{3 a} f_{3}+\cdots+T_{i a} f_{i}$. Then the spectrum distance $(D)$ between the unknown spectra and the average spectrum in the calibration set (Hit) was calculated according to formula (4), and the threshold for each category $\left(D_{T}\right)$ was obtained according to formula (5), where $S_{\text {Dev }}$ is the standard deviation of Hit and $X$ is a coefficient with 0.25 being selected by experience. When $D$ is less than $D_{T}$, the matching degree between the spectra of the unknown sample and calibration samples is high, which suggests the sample spectrum and reference spectra can be considered one category [13]:

$$
\begin{aligned}
D & =\sqrt{\sum_{i}\left[T_{i} a-T_{i} b\right]^{2}}, \\
D_{T} & =\operatorname{Max}(\mathrm{Hit})+X S_{\mathrm{Dev}} .
\end{aligned}
$$

A selectivity parameter $(S)$ is used to determine whether the qualitative model is applicative or not. $S$ is calculated as formula (6). When $S>1$, the classes of 1 and 2 can be completely separated; when $S=1$, the classes can be separated with warning. The larger the $S$ value is, the greater the discrimination result and the accuracy of the prediction model will be [17]:

$$
S=\frac{D}{\left(D_{T 1}+D_{T 2}\right)} .
$$

\section{Results and Discussion}

\subsection{Discrimination of Fritillaries}

3.1.1. Selection of Spectral Pretreatment Method. As shown in Figure 3, the original NIR spectra for different species of Fritillaria are highly similar. The spectrum band between 4000 and $9000 \mathrm{~cm}^{-1}$ is often intercepted for analysis due to its abundant information and high intensity. However, there is severe band overlap, making it difficult to interpret spectral information. Therefore, spectral pretreatment is a critical step in expanding the differences between the spectra of each sample, extracting more effective spectral information, and improving the signal-to-noise ratio.

In this paper, the first derivative method was used for spectral pretreatment, and the scaling to first range method and factorization method were used as the algorithm, respectively. The smoothing point was set at 13 , and spectrum band between 4000 and $5000 \mathrm{~cm}^{-1}$ was selected for analysis.

3.1.2. Establishment of Qualitative Analysis Model. When the factorization method was used as the algorithm, the analysis result showed that $S$ is below 2, indicating that the accuracy of qualitative classification for unknown samples is too low. While the first derivative method was used, the qualitative analysis results of the model were satisfactory (Table 1). Table 1 shows the smallest $S$ values among all species of Fritillaria, and each $S$ value exceeds 2, which suggests that all species of Fritillaria can be completely distinguished. Fourteen Fritillaria samples were used to test the qualitative NIR model. As a result, the mistaken judgment rate of the qualitative NIR model is zero.

\subsection{Quantitative Analysis of the Total Alkaloids from Fritillaria}

3.2.1. Content Determination of the Total Alkaloids by UV Spectroscopy. Acid dye colorimetry has been a commonly used and well-recognized method for the alkaloid content 
TABLE 1: The qualitative analysis results of the model using the first derivative method.

\begin{tabular}{lccc}
\hline Number & Group 1 & ${\text { Group } 2^{\text {a }}}$ \\
\hline 1 & F. thunbergii Miq. (big) & F. pallidiflora Schrenk & 2.208536 \\
2 & F. thunbergii Miq. (small) & F. pallidiflora Schrenk & 10.515240 \\
3 & F. ussuriensis Maxim & F. pallidiflora Schrenk & 3.510903 \\
4 & F. delavayi Franch & F. cirrhosa D. Don & 12.225511 \\
5 & F. unibraacteate Hsiao et. K. C. Hsia & F. pallidiflora Schrenk & 8.895630 \\
6 & F. cirrhosa D. Don & F. delavayi Franch & 12.225511 \\
7 & F. pallidiflora Schrenk & F. thunbergii Miq (big) & 2.208536 \\
\hline
\end{tabular}

${ }^{a}$ Fritillaria types in Group 2 are the most similar varieties to Fritillaria in Group 1, respectively. F. thunbergii Miq. (big) and F. pallidiflora Schrenk are the most similar species to each other, so the $S$ values in number 1 and number 7 are the same (2.208536). F. delavayi Franch and $F$. cirrhosa D. Don are also in the same case.

TABLE 2: Content of total alkaloids from seven Fritillaria samples by UV spectroscopy.

\begin{tabular}{lccc}
\hline Number & Sample & Absorbance & Total alkaloids (\%) \\
\hline 1 & Fritillaria ussuriensis Maxim & 0.282 & 0.1750 \\
2 & Fritillaria thunbergii Miq. (big) & 0.387 & 0.2406 \\
3 & Fritillaria thunbergii Miq. (small) & 0.344 & 0.2138 \\
4 & Fritillaria pallidiflora Schrenk & 0.392 & 0.2430 \\
5 & Fritillaria cirrhosa D. Don & 0.187 & 0.1160 \\
6 & Fritillaria unibraacteate Hsiao et. K. C. Hsia & 0.327 & 0.2030 \\
7 & Fritillaria delavayi Franch & 0.263 & 0.1640 \\
\hline
\end{tabular}

of plants. Its analysis results are used as the true values for quantitative analysis of Fritillaria in this study. Fritillaria alkaloids can form yellow ion pairs with bromothymol blue at $\mathrm{pH} 5$ buffer solutions, and the ion pairs are quantitatively extracted by chloroform. Peiminine is the main component of Fritillaria alkaloids and was therefore selected as the reference compound with the maximum UV absorption of ion pairs in chloroform at $410 \mathrm{~nm}$. The linear regression equation was obtained $(A=0.000541+0.040201 C, r=0.9998)$ with good linearity within the concentration range of $1.78 \sim 8.90 \mu \mathrm{g} / \mathrm{mL}$.

The quantitative analysis results of seven samples were shown in Table 2.

3.2.2. Establishment of NIR Quantitative Model. Based on the pretreated NIR spectra, the NIR quantitative analysis model for the total alkaloids from Fritillaria was established using the PLS method with UV analysis data as the true values.

OPUS software provides two methods for validation of the quantitative model: internal cross validation and external validation. Due to the limited samples used in this experiment, the internal cross validation was used to optimize the model. We used RMSECV values as assessment criteria to acquire the optimal frequency range and PLS components.

The influences of five different PLS spectral pretreatment methods on the NIR quantitative model were listed in Table 3. When Min-Max normalization was adopted, the spectra were shifted linearly, so that the minimum $Y$-value equals zero. Meanwhile the spectra were expanded, so that the maximum $Y$-value equals two absorbance units. This method is comparable to vector normalization. Multiplicative scatter correction is often used for measurements in diffuse reflection, while vector normalization is used to eliminate the influence of different optical path lengths in case of transmission measurements. Subtraction of a straight line is used to eliminate linear baseline shifts, which result from different values of the detector amplification. By calculating the first derivative, a relative enhancement of the sharp structures compared to the original spectrum can be observed and the signals with steep edges get more emphasis than relatively flat bands. Thus the first derivative method can be used to emphasize pronounced but small features compared to huge broad-banded structures and leads to the best calibration results.

The results in Table 1 show that the correlation coefficient $\left(R^{2}\right)$ and RMSECV values by using five different spectral pretreatment methods have little distinction in the optimal frequency range of $6082.8-4165.8 \mathrm{~cm}^{-1}$. In this study, the first derivative was selected as the spectral pretreatment method for the quantitative model.

Optimization of the PLS components was shown in Figure 4. The final results of the model optimization are as follows: PLS spectral pretreatment methods: first derivative method; optimal frequency range: $4165.8 \sim 6082.8 \mathrm{~cm}^{-1}$; optimal PLS components: 8 .

3.2.3. Quantitative Prediction by PLS Model. The established PLS model was used to predict the total alkaloid contents of 28 samples in validation set, and the corresponding prediction values were obtained (Table 4). The values of RMSEP, $R^{2}$, and SEP for the prediction results were $0.00643,0.9893$, and 0.00702 , respectively. To validate the precision of the established NIRS models, SEP should be assessed in relation to the 
TABLE 3: Model verification results by using different spectral pretreatment methods.

\begin{tabular}{|c|c|c|c|c|}
\hline Number & Spectral pretreatment methods & PLS components & $R^{2}$ & RMSECV \\
\hline 1 & Min-Max normalization method & 7 & 0.9944 & 0.00314 \\
\hline 2 & Multiplicative scatter correction method & 6 & 0.9943 & 0.00319 \\
\hline 3 & Vector normalization method & 6 & 0.9942 & 0.00320 \\
\hline 4 & First derivative method & 8 & 0.9935 & 0.00339 \\
\hline 5 & Subtraction of a straight line & 7 & 0.9939 & 0.00378 \\
\hline
\end{tabular}

TABLE 4: Quantitative predictions for the samples in prediction set by PLS model.

\begin{tabular}{|c|c|c|c|c|}
\hline Number & Sample & NIR prediction value $/ \%$ & UV true value $/ \%$ & Deviation \\
\hline 1 & Fritillaria ussuriensis Maxim & 0.1762 & 0.1750 & -0.001240 \\
\hline 2 & Fritillaria ussuriensis Maxim & 0.1757 & 0.1750 & -0.000653 \\
\hline 3 & Fritillaria ussuriensis Maxim & 0.1722 & 0.1750 & 0.002770 \\
\hline 4 & Fritillaria ussuriensis Maxim & 0.1761 & 0.1750 & -0.001110 \\
\hline 5 & Fritillaria thunbergii Miq. (big) & 0.2386 & 0.2406 & 0.002030 \\
\hline 6 & Fritillaria thunbergii Miq. (big) & 0.2454 & 0.2406 & -0.004770 \\
\hline 7 & Fritillaria thunbergii Miq. (big) & 0.2355 & 0.2406 & 0.005060 \\
\hline 8 & Fritillaria thunbergii Miq. (big) & 0.2427 & 0.2406 & -0.002070 \\
\hline 9 & Fritillaria thunbergii Miq. (small) & 0.2110 & 0.2138 & 0.002800 \\
\hline 10 & Fritillaria thunbergii Miq. (small) & 0.2170 & 0.2138 & -0.003230 \\
\hline 11 & Fritillaria thunbergii Miq. (small) & 0.2160 & 0.2138 & 0.002270 \\
\hline 12 & Fritillaria thunbergii Miq. (small) & 0.2099 & 0.2138 & 0.003890 \\
\hline 13 & Fritillaria pallidiflora Schrenk & 0.2416 & 0.2430 & 0.001350 \\
\hline 14 & Fritillaria pallidiflora Schrenk & 0.2383 & 0.2430 & 0.004680 \\
\hline 15 & Fritillaria pallidiflora Schrenk & 0.2416 & 0.2430 & 0.001350 \\
\hline 16 & Fritillaria pallidiflora Schrenk & 0.2513 & 0.2430 & -0.008280 \\
\hline 17 & Fritillaria cirrhosa D. Don & 0.1167 & 0.1160 & -0.0006750 \\
\hline 18 & Fritillaria cirrhosa D. Don & 0.1148 & 0.1160 & 0.001240 \\
\hline 19 & Fritillaria cirrhosa D. Don & 0.1154 & 0.1160 & 0.000615 \\
\hline 20 & Fritillaria cirrhosa D. Don & 0.1188 & 0.1160 & -0.002750 \\
\hline 21 & Fritillaria unibraacteate Hsiao & 0.1967 & 0.2030 & 0.006280 \\
\hline 22 & Fritillaria unibraacteate Hsiao & 0.2079 & 0.2030 & -0.004920 \\
\hline 23 & Fritillaria unibraacteate Hsiao & 0.1978 & 0.2030 & 0.005160 \\
\hline 24 & Fritillaria unibraacteate Hsiao & 0.2071 & 0.2030 & -0.004100 \\
\hline 25 & Fritillaria delavayi Franch & 0.1657 & 0.1640 & -0.001720 \\
\hline 26 & Fritillaria delavayi Franch & 0.1632 & 0.1640 & 0.000758 \\
\hline 27 & Fritillaria delavayi Franch & 0.1637 & 0.1640 & 0.000307 \\
\hline 28 & Fritillaria delavayi Franch & 0.1650 & 0.1640 & -0.001040 \\
\hline
\end{tabular}

precision of the reference method illustrated as the standard error of the laboratory (SEL), and the calculating formulae can be found in [17]. In this work, SEL was calculated to be 0.0051, and SEP was 0.00702 which was less than $2 \times$ SEL, suggesting the precision of the established NIR model was satisfactory for total Fritillaria alkaloid contents analysis. The largest deviation between NIR prediction value and UV true value is $3.3 \%$, which indicates that the established PLS model can give accurate prediction.

The NIR method and the UV method can be compared with a paired $t$-test. In the $95 \%$ confidence interval, the value of statistics $t_{(0.05)}=0.016$ was less than the corresponding critical value $t_{(0.05,27)}=2.052$. The results demonstrated that there was no significant difference between the NIR and the UV method.

3.2.4. Results of Quantitative Analysis by PLS Model. In order to validate the accuracy and applicability of the PLS model, seven kinds of Fritillaria powder samples in the verification set were scanned to acquire NIR spectra. The quantitative prediction values for seven samples were obtained by using established PLS models (Table 5). In the 95\% confidence interval, the value of statistics $t_{(0.05)}=0.020$, which was less than the corresponding critical value, $t_{(0.05,6)}=2.447$, suggests that there was no significant difference between the NIR and the UV method. 
TABLE 5: Quantitative analysis results for the samples in validation set by PLS model.

\begin{tabular}{lccc}
\hline Number & Sample & NIR prediction value/\% & UV true value/\% \\
\hline 1 & Fritillaria ussuriensis Maxim & 0.1728 & 0.1640 \\
2 & Fritillaria thunbergii Miq. (big) & 0.2375 & 0.2030 \\
3 & Fritillaria thunbergii Miq. (small) & 0.2165 & 0.1160 \\
4 & Fritillaria pallidiflora Schrenk & 0.2411 & 0.2430 \\
5 & Fritillaria cirrhosa D. Don & 0.1165 & 0.1750 \\
6 & Fritillaria unibraacteate Hsiao & 0.2080 & 0.2138 \\
7 & Fritillaria delavayi Franch & 0.1661 & 0.2406 \\
\hline
\end{tabular}

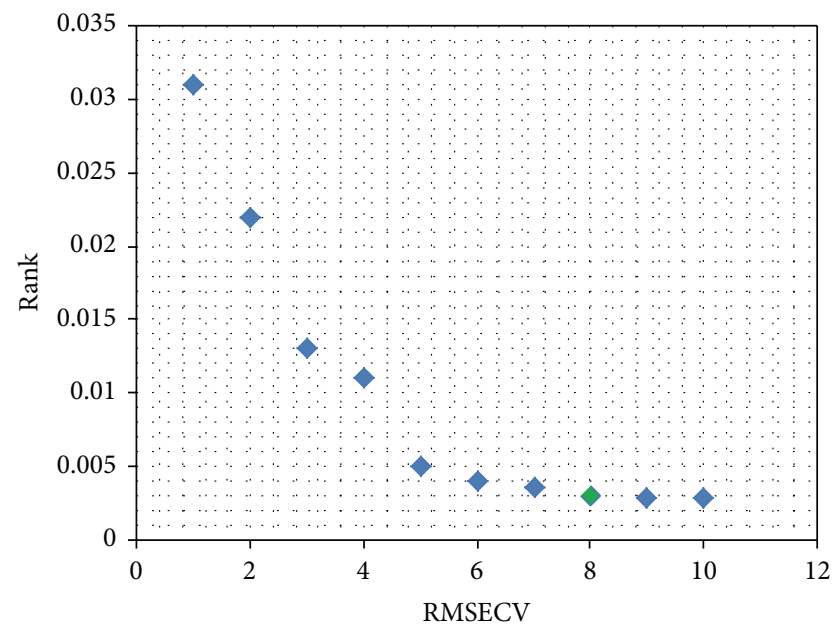

Figure 4: Determination of optimal PLS components by cross validation.

Compared with UV method in quantitative analysis for total alkaloid content of Fritillaria, NIRS method is simpler, faster, and more efficient, due to its nondestructive character. Unlike in the analysis with UV method, the tedious sample treatments were completely avoided in NIR analysis.

\section{Conclusion}

In this study, a qualitative NIR model for discrimination of seven kinds of Fritillaria was established with the first derivative as the spectra pretreatment method. The scaling to first range worked as the algorithm and 4000 to $5000 \mathrm{~cm}^{-1}$ as the scanning wavelength range with 13 points smoothing. The qualitative NIR model was verified with 14 Fritillaria samples. As a result, the mistaken judgment rate of the qualitative NIR model is zero.

A quantitative NIR model for total alkaloids from Fritillaria was established by using the PLS method with UV analysis data as the true values. The first derivative method was used for the spectra pretreatment and the internal cross validation was used to optimize the model. RMSECV values served as assessment criteria to acquire the optimal frequency range and PLS components. The established quantitative NIR model can accurately predict the content of total alkaloids from Fritillaria samples with a correlation coefficient of 0.9935 and a RMSECV of 0.00339 . The paired $t$-test between
NIR prediction values and UV values indicated that there was no significant difference between NIR and UV methods.

As the experiment has shown, the NIR analysis methods developed here provide a new, simple, fast, and accurate approach for the qualitative and quantitative analysis of Fritillaria.

\section{Conflict of Interests}

The authors declare that there is no conflict of interests regarding the publication of this paper.

\section{References}

[1] Y. Tsukamoto, The Grand Dictionary of Horticulture, vol. 4, Shogakukan, Tokyo, Japan, 1989.

[2] O. Genbu and A. Yukio, "Kokai Tokkyo Koho Chinese medicine formulation for cold therapy," Application JP 92$17021219920604,1993$.

[3] J. X. Zhang, A. N. Lao, and R. S. Xu, "Chemical constituents of fresh bulbs of Fritillaria thunbergii Miq," China Journal of Chinese Material Medica, vol. 18, no. 6, pp. 354-382, 1993.

[4] H. N. Ma, "The quantitative analysis alkaloids in Fritillaria unibraacteate Hsiao et. K. C. Hsia and Fritillaria cirrhosa D. Don," China Journal of Chinese Material Medica, vol. 20, no. 3, article 172, 1995.

[5] Q. H. Liu, "The quantitative analysis chemicalcontent in sinkiang Fritillaria," Acta Pharmaceutica Sinica, vol. 19, no. 12, p. 894, 1984.

[6] P. Li, G. Xu, R. Jin $\mathrm{H}$ et al., "Research of traditional Chinese medicine Fritillaria XV. Determination of 21 Fritillaria alkaloids," Journal of China Pharmaceutical University, vol. 21, no. 5, article 319, 1990.

[7] Q. X. Zhang and S. Y. Sha, "Determination of total alkaloids in Fritillaria," Herbal Communications, vol. 7, no. 2, article 13, 1976.

[8] J. Luypaert, D. L. Massart, and Y. V. Heyden, "Near-infrared spectroscopy applications in pharmaceutical analysis," Talanta, vol. 72, no. 3, pp. 865-883, 2007.

[9] D. J. Wargo and J. K. Drennen, "Near-infrared spectroscopic characterization of pharmaceutical powder blends," Journal of Pharmaceutical and Biomedical Analysis, vol. 14, no. 11, pp. 14151423, 1996.

[10] M. Blanco and M. A. Romero, "Near-infrared libraries in the pharmaceutical industry: a solution for identity confirmation," Analyst, vol. 126, no. 12, pp. 2212-2217, 2001.

[11] M. Alcalà, M. Blanco, M. Bautista, and J. M. González, "Online monitoring of a granulation process by NIR spectroscopy," 
Journal of Pharmaceutical Sciences, vol. 99, no. 1, pp. 336-345, 2010.

[12] N. Qu, X. Li, Y. Dou, H. Mi, Y. Guo, and Y. Ren, "Nondestructive quantitative analysis of erythromycin ethylsuccinate powder drug via short-wave near-infrared spectroscopy combined with radial basis function neural networks," European Journal of Pharmaceutical Sciences, vol. 31, no. 3-4, pp. 156-164, 2007.

[13] H. Y. Lu, S. S. Wang, R. Cai, Y. Meng, X. Xie, and W.-J. Zhao, "Rapid discrimination and quantification of alkaloids in Corydalis Tuber by near-infrared spectroscopy," Journal of Pharmaceutical and Biomedical Analysis, vol. 59, no. 1, pp. 4449, 2012.

[14] L. G. Hu, Z. R. Chen, K. Cheng et al., "Rapid quantitative determination of Fritillaria thunbergii Miq mixed into Fritillaria cirrhosa D. Don by near-infrared diffuse reflectance spectroscopy," Chinese Journal of Pharmaceutical Analysis, vol. 25, no. 2, pp. 150-152, 2005.

[15] Y. Gao, Y. F. Chai, and Y. T. Wu, "Identification of Fritillaria by near-infrared diffuse reflectance spectroscopy," Chinese Traditional Patent Medicine, vol. 27, no. 12, p. 1440, 2005.

[16] C.-O. Chan, C.-C. Chu, D. K.-W. Mok, and F.-T. Chau, "Analysis of berberine and total alkaloid content in Cortex Phellodendri by near infrared spectroscopy (NIRS) compared with highperformance liquid chromatography coupled with ultra-visible spectrometric detection," Analytica Chimica Acta, vol. 592, no. 2, pp. 121-131, 2007.

[17] R. Cai, S. S. Wang, Y. Meng, Q. Meng, and W. Zhao, "Rapid quantification of flavonoids in propolis and previous study for classification of propolis from different origins by using near infrared spectroscopy," Analytical Methods, vol. 4, no. 8, pp. 2388-2395, 2012. 

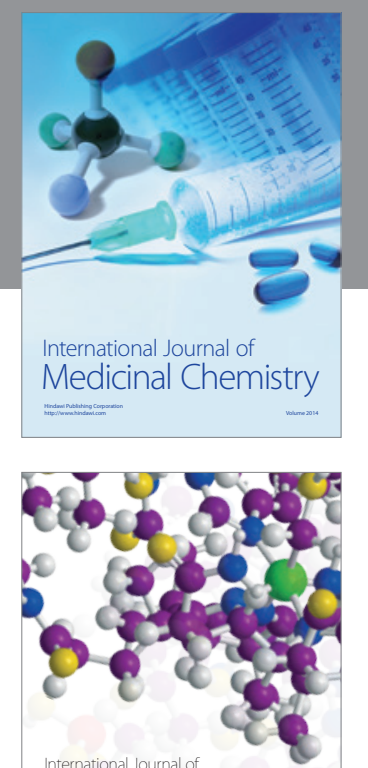

\section{Carbohydrate} Chemistry

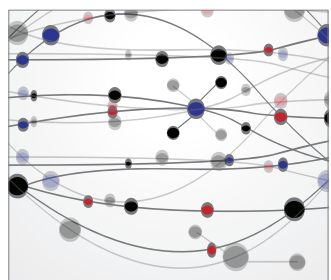

The Scientific World Journal
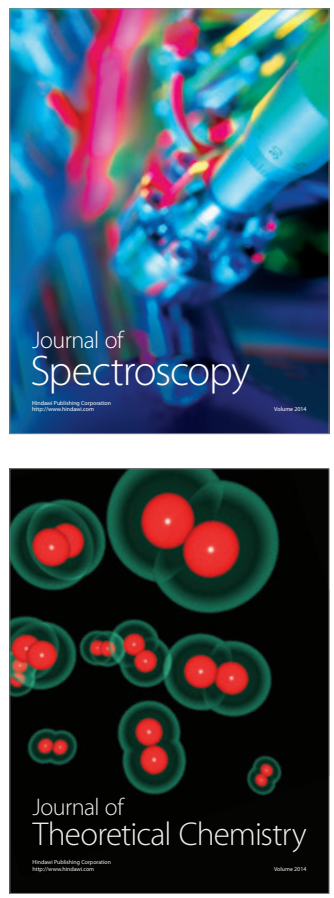
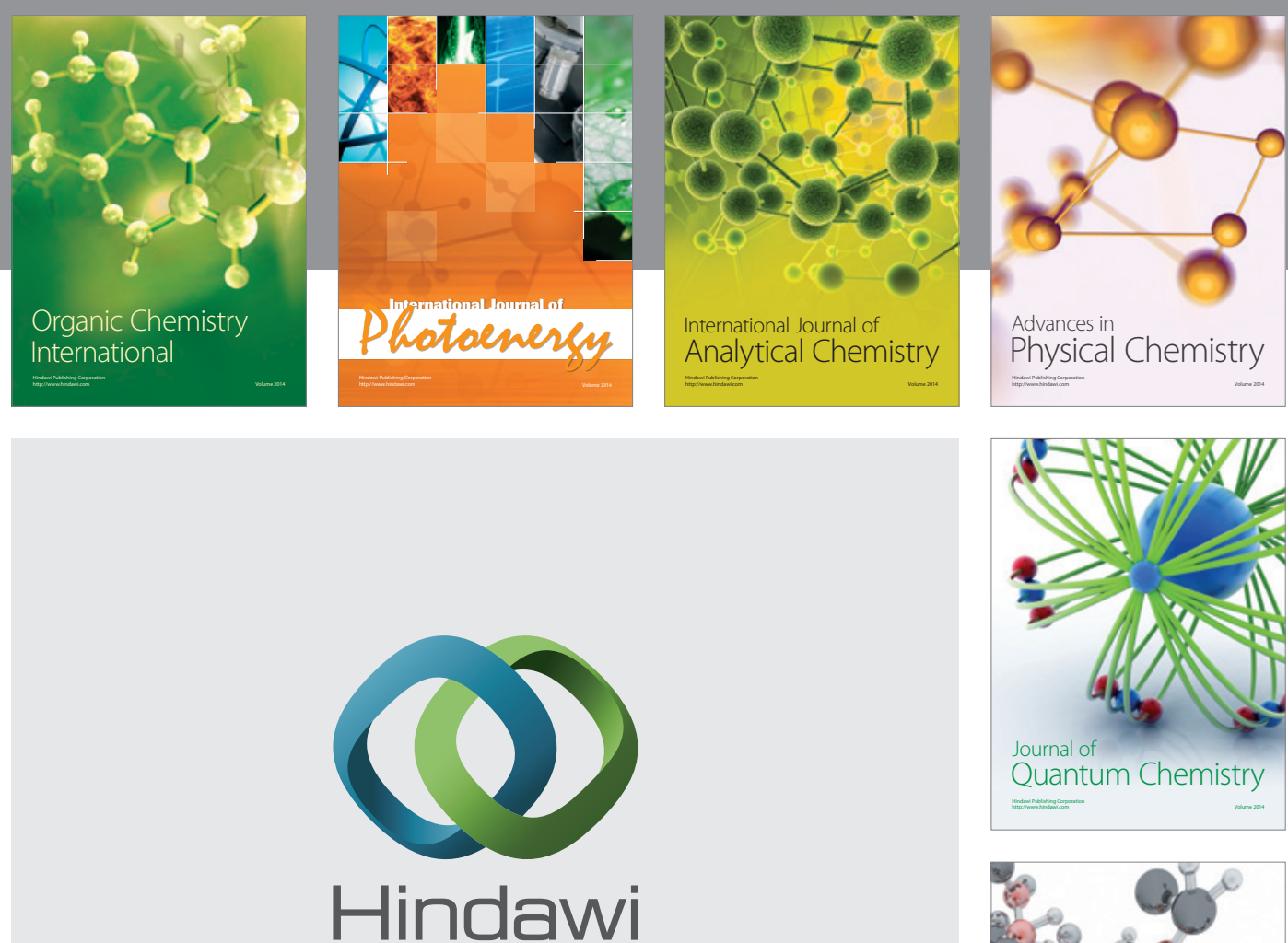

Submit your manuscripts at

http://www.hindawi.com

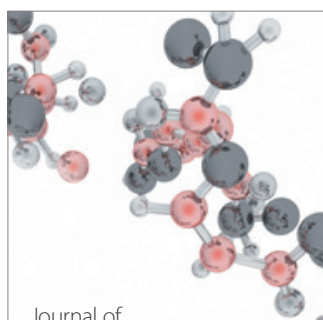

Analytical Methods

in Chemistry

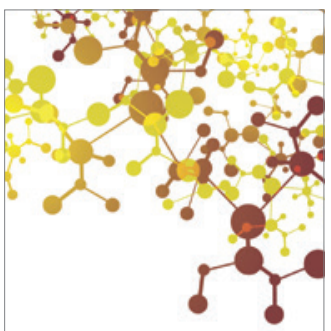

Journal of

Applied Chemistry

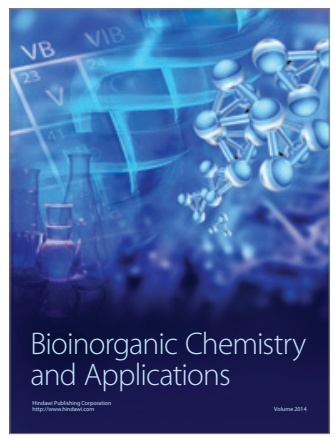

Inorganic Chemistry
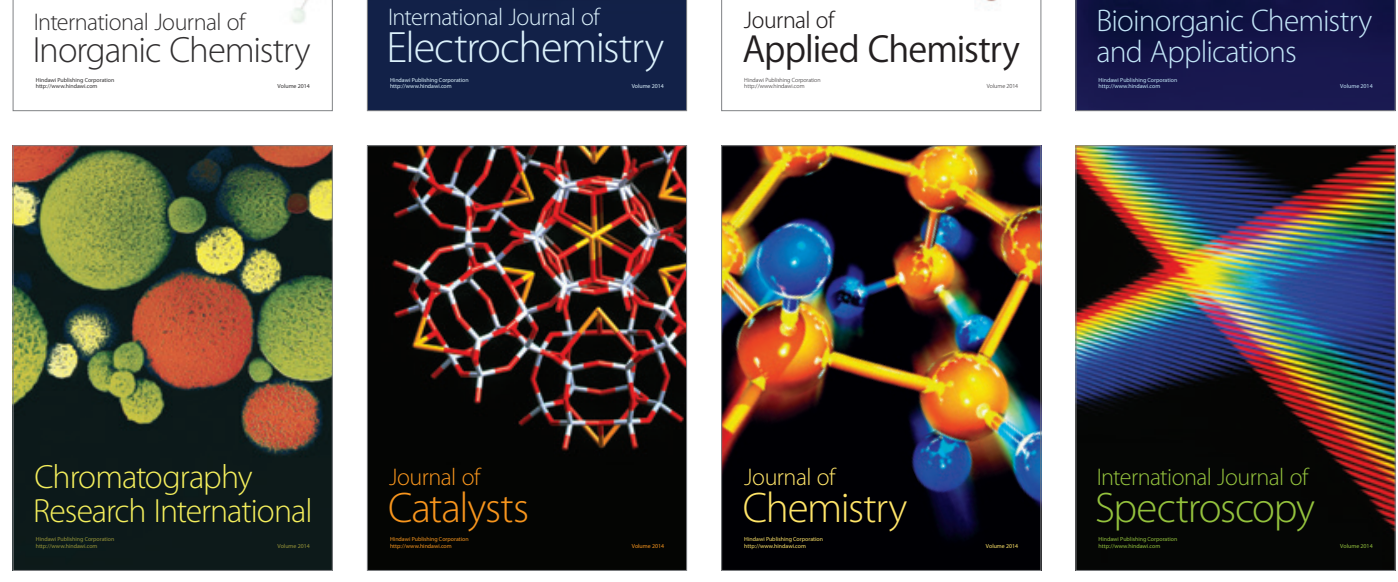